\title{
Defining and Exemplifying Semantic Widgets for Learning
}

\author{
Lars Johnsen and Rocio Chongtay \\ Department of Design and Communication, University of Southern Denmark, Engstien 1, 6000 Kolding, Denmark \\ $\{$ Larsjo,Rocio\}@sdu.dk
}

\begin{abstract}
Keywords: $\quad$ Semantic Widgets, Semantic Technologies, Web of Data, Web 3.0, Metadata, Schema.org, Visual Learning, Infographics.

Abstract: $\quad$ In this article, we make the case for widgets as an appropriate vehicle for interacting with the Web of Data in learning applications and materials. In the article, an attempt is made to define and exemplify the concept of semantic widgets for learning (SW4L) and to suggest how such educational software may be exposed and utilized in the Web of Data. As an example of SW4L, we mention widgets operating on semantic learning content marked up using the web vocabulary of schema.org and illustrate how they may be designed to support instructional guidelines advocating the use of visual aids in learning materials.
\end{abstract}

\section{INTRODUCTION}

As the Web is moving from a Web of Documents to a Web of Data (aka The Semantic Web or Web 3.0), and to a Web of Things, educationalists are beginning to discuss how semantic technologies may be put to use in educational settings and, more narrowly, how learning applications can make use of semantically represented data to enhance learning experiences, (see for instance Carbonaro, 2012).

Ideally, the Web of Data has a great potential for learning, teaching and education. It brings with it the promise of improved discoverability of learning resources, greater reuse of learning content, increased adaptation and personalization of learning materials, more reliable data analytics of user performances, preferences and behaviors, enhanced opportunities for collaboration via social media and so forth.

Palmér (2012) presents some recommendations for building learning applications based on Semantic Web technology and discusses some of the main obstacles found in the process. Other studies explore different combinations of Semantic Web, Web widgets and learning (see Mäkelä et al., 2007 and Soylu et al., 2010). However, it is not altogether obvious how we should go about creating multiplatform "semantic solutions" that can be used to support real learning activities in authentic educational contexts by ordinary teachers and professors. What kinds of applications, or components, are needed and how can these naturally be integrated into existing learning architectures, media and materials?

In this article, we make the case for embedded reusable components as an appropriate vehicle for interacting with semantic content in learning applications and materials. More specifically, we suggest how semantic widgets for learning may be designed and exposed on the Web of Data as shareable educational resources.

In section two, we attempt to define and delimit the concept of semantic widgets for learning. Semantic widgets for learning are described and categorized on the basis of their characteristics as reusable software modules, their functional roles as learning support tools, their capability to operate on structured semantic learning content and, finally, their discoverability on the Web of Data.

In section three, we exemplify the concept of semantic widgets for learning. We do so by introducing one kind of semantic widgets, namely widgets operating on content or data marked up using the widely used web vocabulary of schema.org. We illustrate how such widgets may be designed and developed to support instructional guidelines advocating the use of visual aids in learning materials.

In section four, we suggest how semantic widgets for learning themselves may be annotated to enhance their discoverability and reuse on the Web of Data. Once again, we recommend deploying schema.org categories.

In section five, the conclusions, we briefly argue 
why we think that the approach proposed here is worth pursuing and what implications such an approach may have for the adoption of semantic functionality in learning applications and materials.

\section{SEMANTIC WIDGETS FOR LEARNING - WHAT?}

According to Cáceres (2012), a widget is an interactive single purpose application for displaying and/or updating local data or data on the Web, packaged in a way as to allow a single download and installation on a user's computer or mobile device. Alternatively, a widget may be embedded in a web page to provide additional functionality. These days, widgets are often created using HTML5, CSS and JavaScript to ensure that they can run on different platforms and in a wide range of browsers and other user agents.

Because of their potential for reuse and wide applicability, widgets are becoming popular in educational media, applications and materials, too. One example is iBooks, Apple's format for etextbooks on the iPad. Employing the authoring tool, iBooks Author, e-book writers can embed widgets in their electronic text to provide more engaging learning experiences for their users. These widgets may realize interactive multi-modal curricular content objects, communication tools, exercises, etc. To create a widget, an iBook author may utilize iBooks Author's own built-in widget template, develop his or her own from scratch in HTML5 (possibly using an authoring tool) or configure and download a widget from Bookry.com, one of several websites offering templates for the construction of widgets for iBooks.

Thus, a widget for learning may be defined as an embeddable software module designed to support one or a small number of learning activities. A widget for learning might:

- convey subject matter

- facilitate comprehension of subject matter

- help learners search, retrieve, explore and organize content

- enable learners to communicate, share knowledge or create signs of learning

- facilitate simulation or skills practice

- analyse a relevant data set

- test or evaluate specific learning outcomes

- compensate learners' disabilities

Widgets for learning may further be divided into subclasses depending on the learning theory they support or the instructional design principle(s) they seek to instantiate. For example, a widget manipulating visual learning content to facilitate the comprehension of subject matter may, explicitly or implicitly, be designed to implement one or more of Mayer's principles of multimedia learning, (Mayer, 2009) or Carney and Levin's "10 tenets for teachers", (Carney and Levin, 2002).

Conceptually, widgets for learning are similar to learning objects. One popular definition of learning object is this one:

A Learning Object is an independent and selfstanding unit of learning content that is predisposed to reuse in multiple instructional contexts, (Polsani, 2003).

Both learning objects and widgets for learning are, in other words, digital educational resources created with reuse in mind. Although the boundary between the two notions is somewhat fluid, it may be argued that widgets for learning primarily provide interactive functionality to support learning activities and processes while the main role of learning objects normally is to convey subject matter.

A semantic widget for learning (SW4L) may generally be defined as a widget for learning designed to extract, display or manipulate "semantically structured data" typically, but not invariably, exposed on the Web of Data. Characteristically, these data:

- contain global identifiers to denote entities, and types of entities, on the Web,

- utilize syntaxes like RDF/XML, Microdata, RDFa (Lite) or JSON-LD,

- point to descriptive categories belonging to publicly available vocabularies on the Web such as schema.org, SKOS, FOAF, or ALOCOM,

- contain typed links to connect entities across data sets.

\section{SEMANTIC WIDGETS FOR LEARNING - ONE EXAMPLE}

To exemplify the concept of SW4L, one may mention semantic widgets designed to function on digital resources marked up using the web vocabulary of schema.org (http://schema.org). This is a set of descriptive categories, properties and relations devised and supported by the three major search engines, including Google's. Schema.org offers a wide range of options for semantically enriching digital resources (for learning): Firstly, it may be deployed to add traditional metadata to 
resources, in schema.org parlance "Creative Works", be they textual, visual or audial (books, articles, images, videos, sound bites, etc.). Secondly, it provides some means for encoding information about the subject matter of these resources in the form of types, properties and actions ( $\mathrm{X}$ is a kind of $\mathrm{Y}$; $\mathrm{X}$ has property $\mathrm{Z}$; $\mathrm{X}$ does $\mathrm{A}$ to $\mathrm{B}$ at place $\mathrm{C}$ ). Thirdly, it supports Linked Data mechanisms to create pointers into external learning object metadata schemes such as ALOCOM or web-based data sets like Freebase or DBpedia. Last but not least, the schema.org vocabulary is compatible with several markup syntaxes, currently Microdata, RDFa (Lite) and JSON-LD. This means that schema.org metadata can be applied to many forms of digital content: it can be embedded in online web pages as well as off-line e-books since the most recent version of the EPUB format (3.01) supports Microdata and RDFa (Lite); it can be encoded in running text or embedded in two-dimensional graphics provided that the format is SVG.

To illustrate how SW4L's based on schema.org metadata may work to achieve instructional design goals, we are developing some reusable widgets in HTML5/CSS/JavaScript within a use case context. These widgets are meant to provide learners access to infographics, multi-modally presented content, about the topic they are studying.

The use of infographics, especially advance and graphical organizers (see below), in instructional materials has been extensively discussed in the literature and their usefulness as learning support tools empirically tested and evaluated, (see for instance Ausubel, 1978, Carney and Levin, 2002 and Mayer, 2009).

On the surface, a semantic widget for learning (based on schema.org input) may look like a traditional piece of educational software. The difference is, however, that such widgets are supposed to provide more standardized, and therefore more reusable, metadata-driven designs. A semantic widget generating, say, a visual timeline on the basis of data distilled from a web page marked up using the schema.org category of "http://schema.org/Event" will do the same to any text containing data of this type.

\subsection{Use Case - Generating Graphic Organizers}

One use case involving the use of infographics in learning designs is centred round graphic organizers, visual reading adjuncts designed to promote identification, comprehension, recall and retention of conceptual or narrative structure in running text, (see Stull and Mayer, 2007). Graphic organizers comprise synoptic visuals such as tree structures, matrices, charts, diagrams, concept maps and flow charts usually placed in close proximity to the text they are to represent.

Normally, such aids are "hardwired" into the material as embedded static resources. More dynamic, engaging and customized graphic organizers can be envisaged, however, if widgets are allowed to operate on semantic data structures consistently marked up inside, or linked to, the text itself. Metaphorically, these data structures may be conceived of as a kind of latent "semiotic enzymes". They lie dormant in the learning material ready to trigger text transformation processes of various types, for example visualization. And if the text contains typed links to external data sets, subsets of these data may be extracted and added to the visualization output to enhance or elaborate its communicative purpose.

Visualizations like graphic organizers may be interactively controlled: A learner may choose to see the text's key concepts as a network diagram beside the text or as a transparent overlay with lines connecting the individual concepts in the text. He or she may be able to control a slider permitting $\mathrm{him} /$ her to incrementally "gray out" less important parts of the text; or he/she may be able to fold the

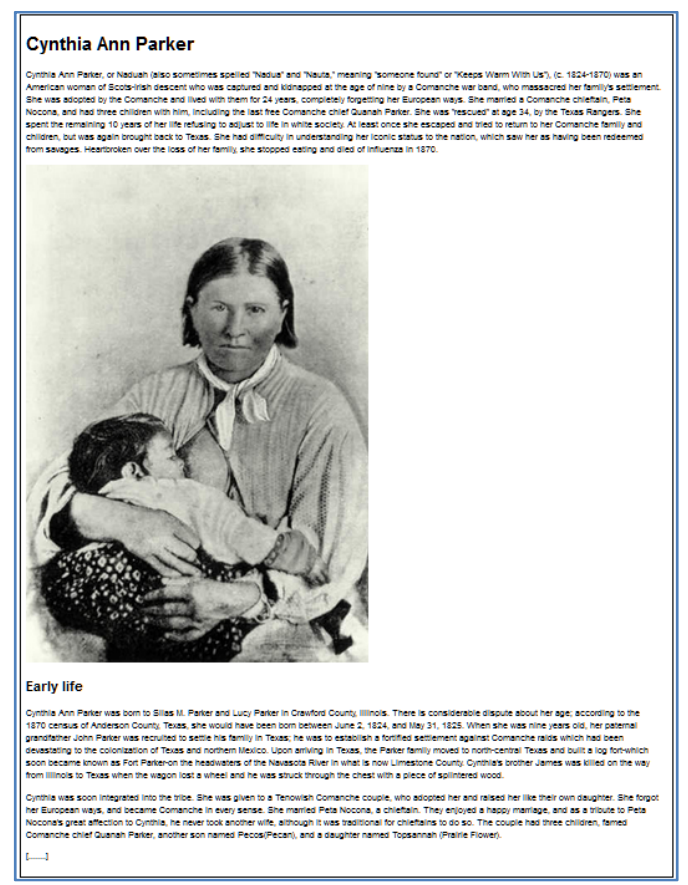

Figure 1: Example of text being reviewed by a learner: a snippet of "Cynthia Ann Parker's" biography from Wikipedia. 
text in and out to display chronological sequences of events or actions in the underlying narrative structure.

Such options can obviously aid learners of different learning styles but also more generally provide multimodal means of foregrounding or reiterating central meanings in the domain being described, typically concepts, facts, procedures or rules. Semiotically speaking, the semantic widgets perform transduction, i.e. remake or reshape meaning in different semiotic modes: text, color, typography, spatial position, etc., (see Bezemer and Kress, 2008). In so doing, they will bring to the fore certain semantic aspects of the text while subduing others.

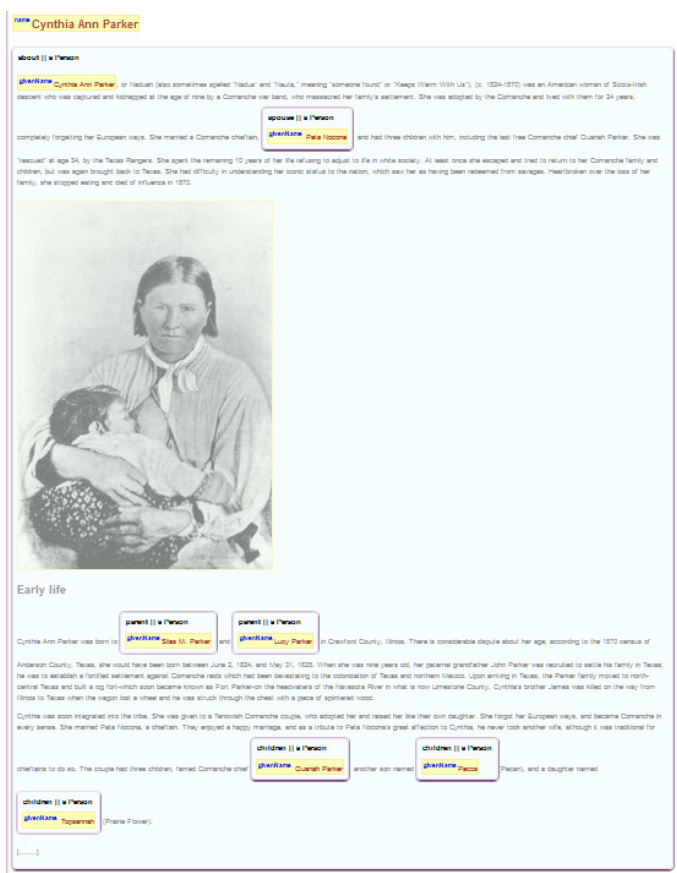

Figure 2: Graphic organizer of the text in Figure Figure 1. Here the marked up text of persons and their relations is highlighted while the rest of the text is grayed out.

\section{spouse [ a Person$$
\text { givenName Peta Nocona }
$$

Figure 3: Detail from the highlighted text in Figure 2.

As more and more visual design tools, script libraries and programming environments see the light of day, more, and increasingly sophisticated, infographics for learning may be developed - and with less effort. To emphasize this point, we have created the graphic organizers shown in the figures in this paper with publicly available tools:

- The styling shown in Figure 2 has been made using rdfa-lab (Niklas Lindström (c) 2012). (https://github.com/niklasl/rdfa-lab)

- The node graph in Figure 4 has been built using D3.js javascript library, based on Mike Bostock's Collapsible Tree.

(http://bl.ocks.org/mbostock/4339083)

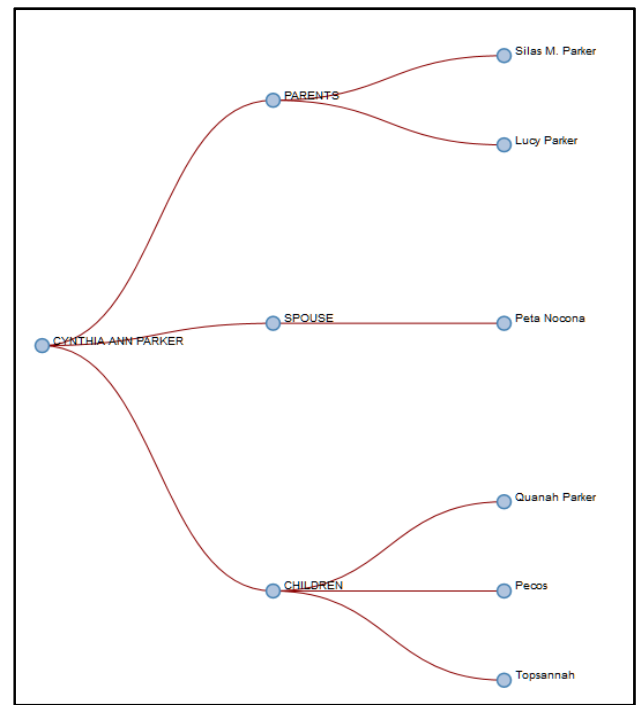

Figure 4: This graphic organizer is representing personal relations in the text in Figure 1 as a graph.

\section{SEMANTIC WIDGETS FOR LEARNING - HOW TO ANNOTATE AND EXPOSE THEM?}

Needless to say, semantic widgets for learning are pretty useless as reusable resources if they cannot be efficiently discovered and retrieved from the Web and effectively evaluated as educational software by potential users. This in turn will only be possible if the widgets contain relevant metadata about themselves as educational resources and if these metadata are represented in formats that can be consumed by search engines and similar software tools. Semantic widgets should take their own medicine, as it were!

We propose that three types of metadata be attached to semantic widgets for learning:

Firstly, they should be described in terms of their characteristics as software applications (e.g. application category, file format, system or device requirements, version number, feature list, 
installation details, etc.). Secondly, information about their function as learning resources should be provided (e.g. instructional goal, intended audience, subject, topic, learning scenario or educational setting, underlying instructional theory, etc.). Thirdly, it should be made explicit what data sets they operate, or are expected to operate, on (e.g. URL, format, descriptive vocabulary, etc.)

To endow semantic widgets for learning with technical, pedagogical/didactical and "data target" metadata, we recommend once more employing the schema.org vocabulary. There are several reasons for this:

- Schema.org is supported by the three major search engines. This is likely to mean enhanced discoverability and improved presentation of search results in the (near) future but no doubt also the availability of more and better tools for tagging and annotation.

- Schema.org contains descriptive categories and properties to annotate all three types of metadata recommended above: To describe semantic widgets as software, the category of SoftwareApplication and its properties may be applied. To indicate what data is at play, the categories of Dataset, DataCatalog and DataDownload are available. And, finally, to designate instructional characteristics, selected properties of the CreativeWork type (e.g. Article or Book) come in handy. Originally, schema.org did not offer labels to tag educational (web) content. But recently schema.org has adopted a set of categories from The Learning Resource Metadata Initiative, LRMI, (http://www.lrmi.net) allowing publishers and others to annotate educational resources and, equally importantly, to align these resources with existing external educational frameworks and standards (e.g. LOM and Common Core State Standards).

- Schema.org can then function as a "one-stop shop" for publishers of semantic widgets for learning who only need to go to one place when looking for appropriate categories for description and annotation.

- Schema.org types and properties are, as already said, compatible with all major data formats associated with Linked Data and Web 3.0 (Microdata, RDFa Lite, JSON-LD).

\section{CONCLUSIONS}

Now, what are the potential benefits of semantic widgets for learning? We think there may be a few:
As mentioned, semantic widgets necessarily entail a more standardized, and therefore more reusable, way of operating on data.

Semantic widgets may be embedded in a wide range of learning applications and materials, including e-textbooks, web pages and so on. That is to say, we can incorporate, or "plug in", limited semantic functionality in otherwise traditional educational resources. This, we think, will significantly lower the barrier to the Web of Data in authentic learning contexts and settings.

Since semantic widgets for learning are small(ish) and modular in terms of functionality, and hence code, they can be produced fairly easily and cheaply and in a piecemeal fashion. And if semantic widgets for learning are produced and distributed under Creative Commons and/or Open Source licenses, there is no reason why, in due course, we should not see a thriving "home industry" in this area. For example, one may envisage web sites similar to Bookry.com specializing in semantic widgets for specific publication channels like interactive e-textbooks based on EPUB 3.01.

Semantic widgets may be linked to specific data sets to form "live" learning objects, that is to say interactive, multimodal dynamic learning units drawing on different resources on the Web of Data. Again, this can be done using the schema.org vocabulary, which contains categories for describing and linking to downloadable data sets anywhere on the Web.

Last, but not least semantic widgets for learning have generally the potential to support what has elsewhere been dubbed "Learning Content Design as a Service" (see Johnsen \& Hansen, 2013). The fundamental idea is that consistently structured, i.e. semantically encoded, content for learning can be freely linked to semantic widgets on the web creating richer and more engaging educational resources.

\section{REFERENCES}

Ausubel, D., 1978. In defense of advance organizers: $A$ reply to the critics. Review of Educational Research, 48, 251-257.

Bezemer, J \& Kress, G., 2008. Writing in Multimodal Texts. A Social Semiotic Account of Designs for Learning, Written Communication, 2008, 25:166.

Carbonaro, A., 2012. Interlinking e-Learning Resources and the Web of Data for Improving Student Experience. Journal of e-Learning and Knowledge Society - English Version, Vol 8, No 2.

Cáceres, M. (ed.). Packaged Web Apps (Widgets) - 
Packaging and XML Configuration (Second Edition). W3C Recommendation 27 November 2012. http://www.w3.org/TR/2012/REC-widgets-20121127/.

Carney, R. N. \& Levin, J. R., 2002. Pictorial Illustrations Still Improve Students' Learning From Text. Educational Psychological Review, vol. 14, no. 1, pp. 5-26.

Johnsen, L. \& Hansen, J. J., 2013. Progressive Semiotic Enrichment: Designing Learning Content Metadata for Web 3.0. In Foley, O., Restivo, M. T., Uhomoibhi, J. \& Helfert, M. (eds.): CSEDU 2013. Proceedings of the 5th International Conference on Computer Supported Education. SciTePress - Science and Technology Publications.

Mäkelä, E., Viljanen, K., Alm, O., Tuominen, J., Valkeapää, O., Kauppinen, T., Kurki, J., Sinkkilä, R., Känsälä, T., Lindroos, R., Suominen, O., Ruotsalo T., and Hyvönen E., 2007. Enabling the Semantic Web with Ready-to-Use Web Widgets. Proceedings of the First Industrial Results of Semantic Technologies Workshop, ISWC2007, November 11, 2007.

Mayer, R. E., 2009. Multimedia Learning. Cambridge University Press, 2nd edition.

Palmér, M., 2012. Learning Applications based on Semantic Web Technologies. Ph.D. thesis, KTH Department of Information Technology, Uppsala University, Sweden.

Polsani, P. R., 2003. Use and Abuse of Reusable Learning Objects. Journal of Digital Information, Vol. 3, No. 4.

Soylu, A., Wild, F., Mödritscher, F., De Causmaecker, P., 2010. Towards Developing a Semantic Mashup Personal and Pervasive Learning Environment: SMupple. In: Proceedings of the 3rd Workshop on Mashup Personal Learning Environments (MUPPLE 2010), CEUR-WS.org, Barcelona, Spain, 2010.

Stull, A. T. and Mayer, R. E. 2007. Learning by Doing Versus Learning by Viewing: Three Experimental Comparisons of Learner-Generated Versus AuthorProvided Graphic Organizers. Journal of Educational Psychology Copyright 2007 by the American Psychological Association, Vol. 99, No. 4, 808-820. 